\title{
Diseño y Construcción de un Prototipo de Electrocardiografía Dinámica "Holter" Universal de Tres Canales con Interfaz Electrónica para Cualquier Plataforma de Instrumentación Virtual
}

\section{Design and Construction Of a Prototype Universal Dynamic Electrocardography "Holter" of Three Channels With Electronic Interface for Any Virtual Instrumentation Platform}

\author{
Quintero M. Jorge E.', Sierra M. José M.² \\ ${ }^{1}$ Facultad de Ingeniería Biomédica, Universidad Manuela Beltrán, Bucaramanga, Colombia, \\ jorge.quintero@umb.edu.co \\ 2 Facultad de Ingeniería Biomédica, Universidad Manuela Beltrán, Bucaramanga, Colombia,
}

jmauricio05@gmail.com

Recibido: 12/08/2012 • Aprobado.15/11/2012

\section{RESUMEN}

Este artículo describe el diseño y la construcción de un electrocardiógrafo portátil (Holter) para la adquisición y el almacenamiento de la primera y segunda derivación bipolar ECG, y la derivación precordial. El prototipo construido tiene la ventaja sobre equipos comerciales similares, que descarga las señales almacenadas en forma analógica, lo que permite utilizar cualquier plataforma de instrumentación virtual, entre otras, Labview y Biopac, ya que no dependen de protocolos de comunicación. Las señales de salida del Holter, se compararon con las entradas proporcionadas por un simulador de paciente ECG, donde se encontró una gran similitud en las amplitudes, formas y espectro de frecuencia, sin que se variara el ritmo cardiaco, la señal de interés que el cardiólogo ha de estudiar, en caso de pacientes con arritmias.

Palabras clave: arritmias, derivaciones ECG, electrocardiograma, holter, instrumentación virtual

\section{AbstRact}

This paper describes the design and construction of a portable electrocardiogram (Holter) for the acquisition and storage of the first and second bipolar derivation ECG and precordial lead. The prototype built has the advantage over similar commercial equipment, that downloads the stored signals in analog form, which lets you use any virtual instrumentation platform, such as LabVIEW, BIOPAC or otherwise, and does not rely on communication protocols. The output signals of the Holter, were compared with the input provided by an ECG patient simulator, where he found a great similarity in the amplitudes, shapes, and frequency spectrum without varying the heart rate, the signal of interest study by cardiologist in case of patients with arrhythmias.

Keywords: arrhythmias, electrocardiogram, holter, lead ECG, virtual instrumentation 


\section{INTRODUCCIÓN}

Un equipo Holter de electrocardiografía (ECG) se utiliza para la monitorización ambulatoria del registro de las señales eléctricas producidas por la actividad cardiaca, durante un tiempo amplio, generalmente de 24 a 48 horas, en una persona que está en movimiento; es decir, desarrollando sus actividades normales o cotidianas. El examen consiste en la instalación de un equipo de registro continuo de 2 o 3 derivaciones ECG, que posteriormente se revisa en un sistema lector, generalmente en la pantalla de un computador. El paciente debe consignar los síntomas que presenta durante el tiempo que permanece con el equipo Holter conectado mediante los electrodos de ECG. Esto permite relacionar el síntoma consignado con el ritmo en ese período. Además, mediante programas computacionales, el sistema lector puede identificar automáticamente los períodos de frecuencia cardíaca mayor y menor, los períodos de ritmo irregular, los cambios en la configuración del complejo QRS, etc., [1].

Existen dos razones fundamentales que hacen de un sistema de monitoreo ambulatorio o prueba de Holter un método básico de exploración cardiovascular: la primera consiste en que determinadas patologías no pueden ser diagnosticadas utilizando métodos convencionales de electrocardiografía, debido a la ausencia de correlación entre la sintomatología aquejada por el paciente y los hallazgos electrocardiográficos en reposo $\mathrm{y}$, la segunda, la información extraída desde el entorno propio del paciente a lo largo de una jornada habitual y con ausencia absoluta de riesgo ya que constituye un método no invasivo de exploración [2].

- El empleo de un Sistema de Monitoreo Ambulatorio se utiliza principalmente para:

- Detección de eventos eléctricos relacionados con el pronóstico de la enfermedad cardiaca [3].

- El diagnóstico, control y seguimiento de la cardiopatía isquémica [4].
- Diagnóstico de arritmias o trastornos de conducción.

- Documentación de la eficacia terapéutica de agentes anti-arrítmicos y anti-isquémicos [5].

- Seguimiento de pacientes con marcapasos.

\section{Diseño del Equipo Holter}

El prototipo del equipo Holter electrocardiográfico se diseña para la adquisición de las derivaciones bipolares DI y DII y una cualquiera de las seis derivaciones precordiales Vx. Una condición del diseño propuesto, consiste en que las señales almacenadas al descargarlas a la computadora lectora se presenten en forma analógica, para que se pueda utilizar cualquier sistema de instrumentación virtual, evitándose así el tener que utilizar un determinado protocolo de comunicación.

\section{A. Etapa de procesamiento análogo de las señales ECG}

La Fig. 1, presenta el diagrama de bloques de la etapa de procesamiento análogo de las señales o derivaciones ECG.

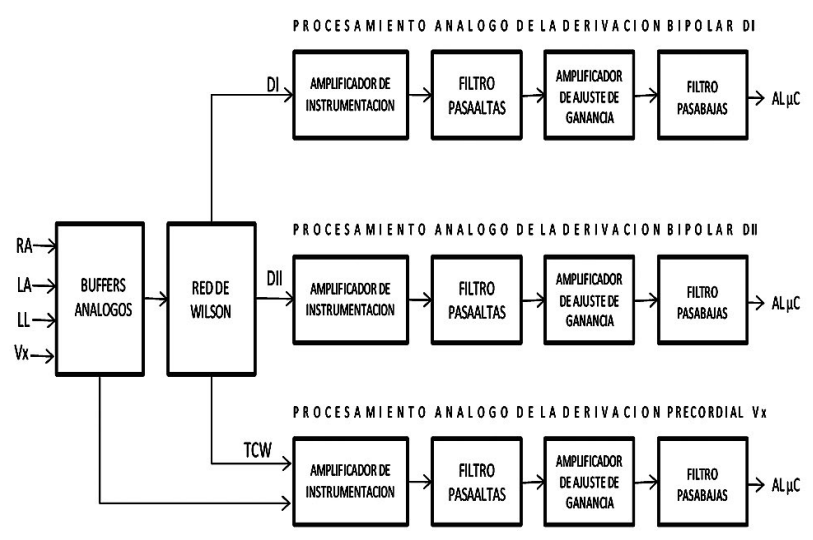

Fig. 1 Diagrama de bloques del procesamiento análogo de las derivaciones ECG. 
Como puede observarse, se diseñaron tres canales de procesamiento análogo para las señales ECG, debido a que en Electrocardiografía Dinámica, se requiere correlacionar las tres derivaciones al mismo tiempo; por lo tanto, no se empleó multiplexación,, que hubiera permitido utilizar un solo canal.

Se emplearon 4 buffers análogos, como acopladores de impedancia, con ganancia de tensión unitaria, para acoplar la alta impedancia de la interface electrodo piel con la baja impedancia de la red de Wilson. La Fig. 2 muestra el banco de buffers análogos.

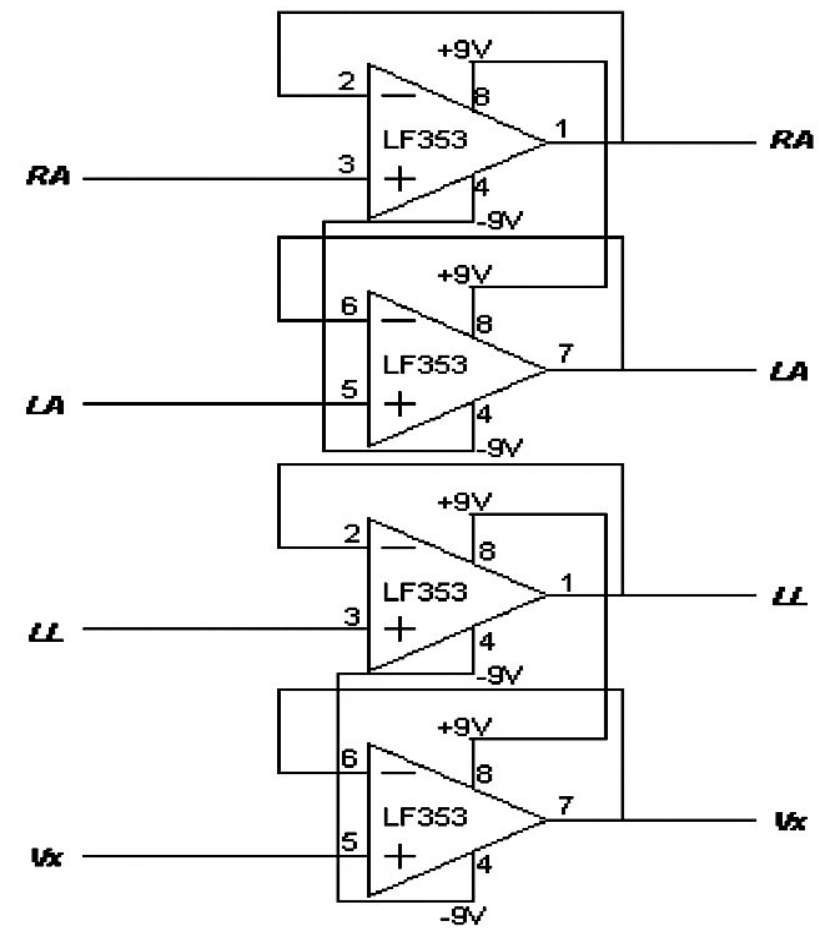

Fig. 2 Banco de Buffers Análogos

Para obtener el potencial promedio aritmético (medido con respecto a tierra) de las tres señales de las extremidades activas (brazo derecho, brazo izquierdo y pierna izquierda), se utilizó la clásica red de Wilson, denominada Potencial del Terminal Central de Wilson (WCT), requerida para la adquisición de la derivación precordial Vx Fig. 3.

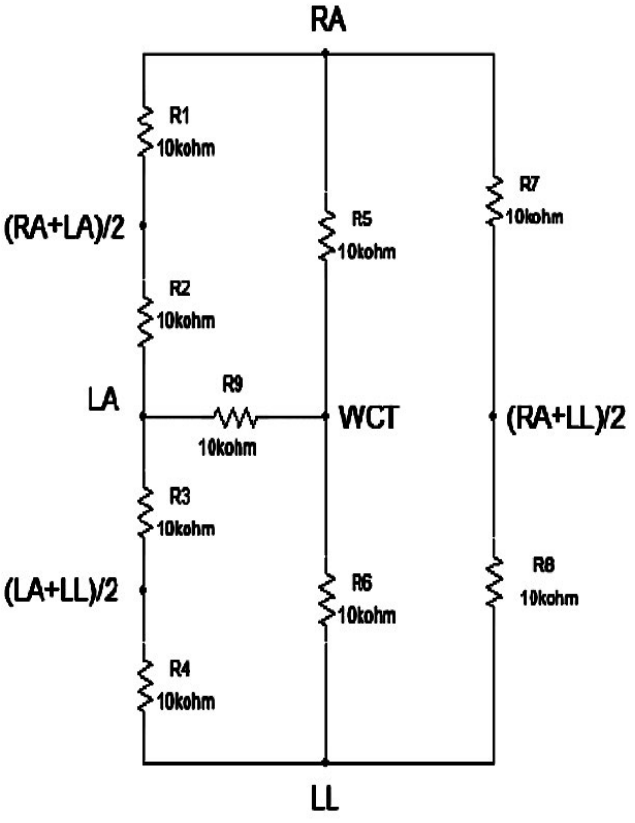

Fig. 3 Red de Wilson

De la Red de Wilson, se tomaron los potenciales de las extremidades activas para ser amplificadas por los Amplificadores de Instrumentación con ganancia de 10, y así evitar la saturación de los mismos por las tensiones DC introducidas por los potenciales de semicelda generados por los electrodos.

Cada una de las señales de salida de los amplificadores de instrumentación se pasan por un filtro pasaaltas Buttherwort con frecuencia de corte de $0.05 \mathrm{~Hz}$ y atenuación de $20 \mathrm{~dB} / \mathrm{dec}$, con el fin de eliminar cualquier componente DC, que pueda saturar los amplificadores de ajuste. Estos amplificadores se diseñaron con ganancia de 100 para que la amplificación total del canal fuera de 1000, como lo establece la norma para electrocardiografía.

La Fig. 4 muestra el Amplificador de Instrumentación, el Filtro Pasaaltas, el Amplificador de Ajuste de Ganancia y el circuito de protección de pierna derecha $(R L)$, correspondiente a uno de los tres canales ECG. Se utilizó el INA114 como Amplificador de Instrumentación y el circuito integrado TL084 que contiene 4 Amplificadores Operacionales para los circuitos de filtrado y ajuste de ganancia. 


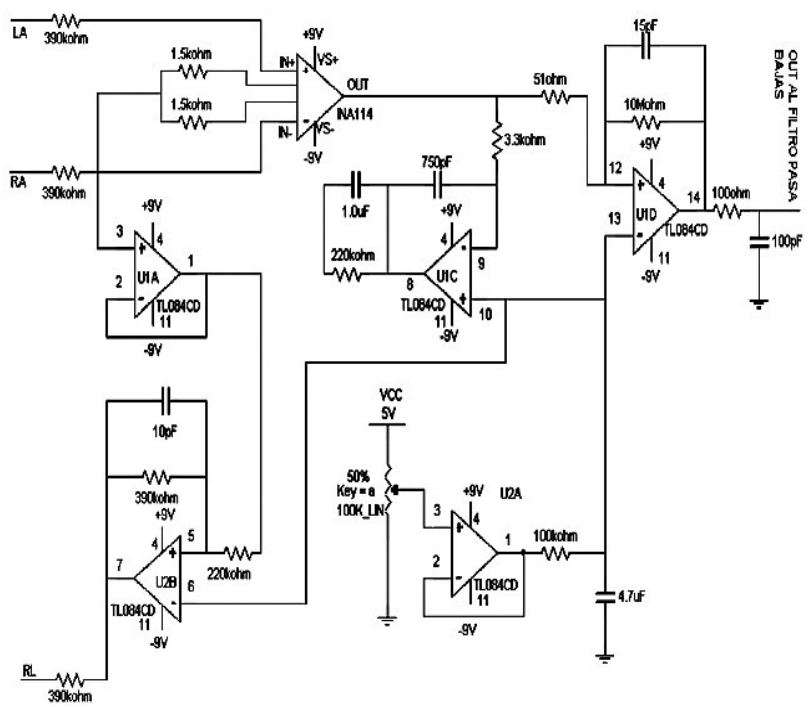

Fig. 4 Amplificador de Instrumentación, Filtro Pasaaltas, Amplificador de Ajuste de Ganancia y Circuito de Protección de Pierna Derecha

Finalmente, las derivaciones amplificadas se pasan por filtros pasabajas Buttherwort con frecuencia de corte de $40 \mathrm{~Hz}$ y atenuación de 160 dB/dec., para eliminar cualquier ruido de $60 \mathrm{~Hz}$ y poder entregar una señal limpia a los convertidores $A / D$ del microcontrolador. La Fig. 5 muestra el filtro pasabajas diseñado.

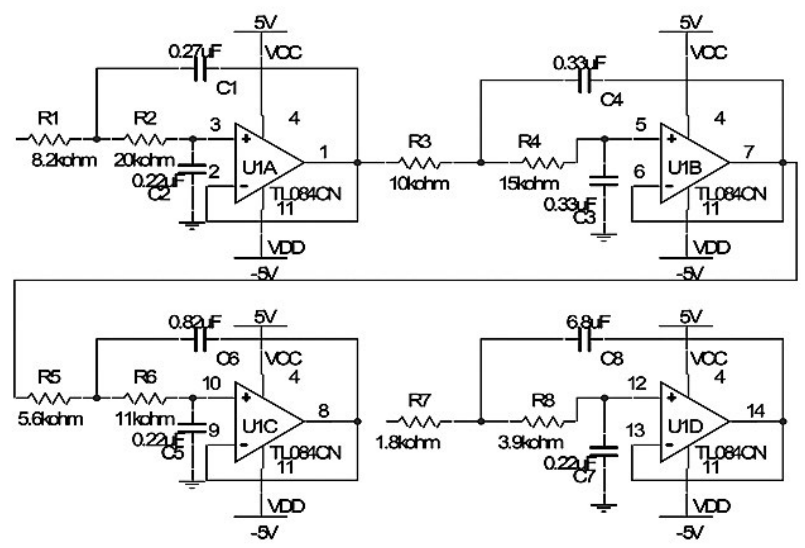

Fig. 5 Filtro Pasabajas de $40 \mathrm{~Hz}$ y $160 \mathrm{~dB} / \mathrm{dec}$.

\section{B. Etapa de procesamiento digital de las señales ECG}

Se utilizó el microcontrolador PIC16F877A, circuito integrado programable de 40 pines, 5 puertos de entrada/salida, 50 interrupciones de manejo, 8 canales de entrada análogo/digital y un puerto esclavo paralelo.

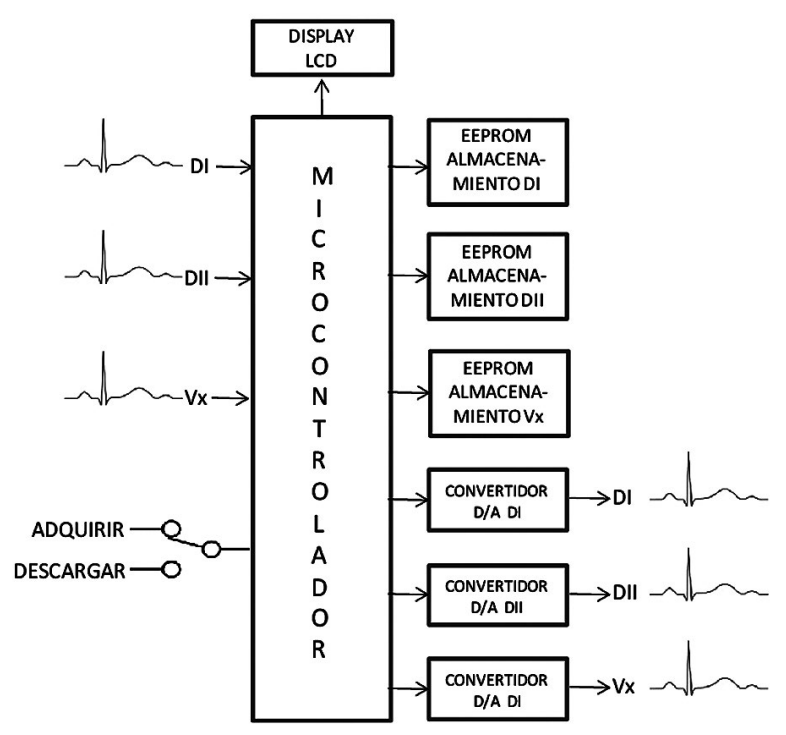

Fig. 6 Diagrama de bloques del procesamiento digital de las derivaciones ECG.

Para almacenar digitalmente cada una de las derivaciones adquiridas, se utilizaron memorias EEPROM, dispositivos de almacenamiento no volátil, que pueden ser borradas eléctricamente. Son de tamaño reducido, se pueden montar sobre un mismo bus de datos y no necesitan tensión negativa para funcionar. Debido a estas característica se optó por trabajar con tres memorias 24LC512, una por cada canal.

Para la conversión digital-análoga de los datos se seleccionó el conversor D/A de 8 bits DAC0832 principalmente porque permite tener una interfaz directa con el microcontrolador. Este dispositivo es de mediana velocidad, con un solo canal, de interface paralela, de baja potencia $(20 \mathrm{~mW})$ y trasforma una entrada digital a una salida analógica en alrededor de $1.0 \mu$ seg.

Se utilizó una pantalla LCD para la comunicación hombre-máquina.

La Fig. 7 muestra el algoritmo de la programación del microcontrolador; es decir, los procesos de adquisición y almacenamiento de las tres derivaciones o señales ECG, la descarga al computador lector y el reseteo o borrado de los bancos de memoria. 


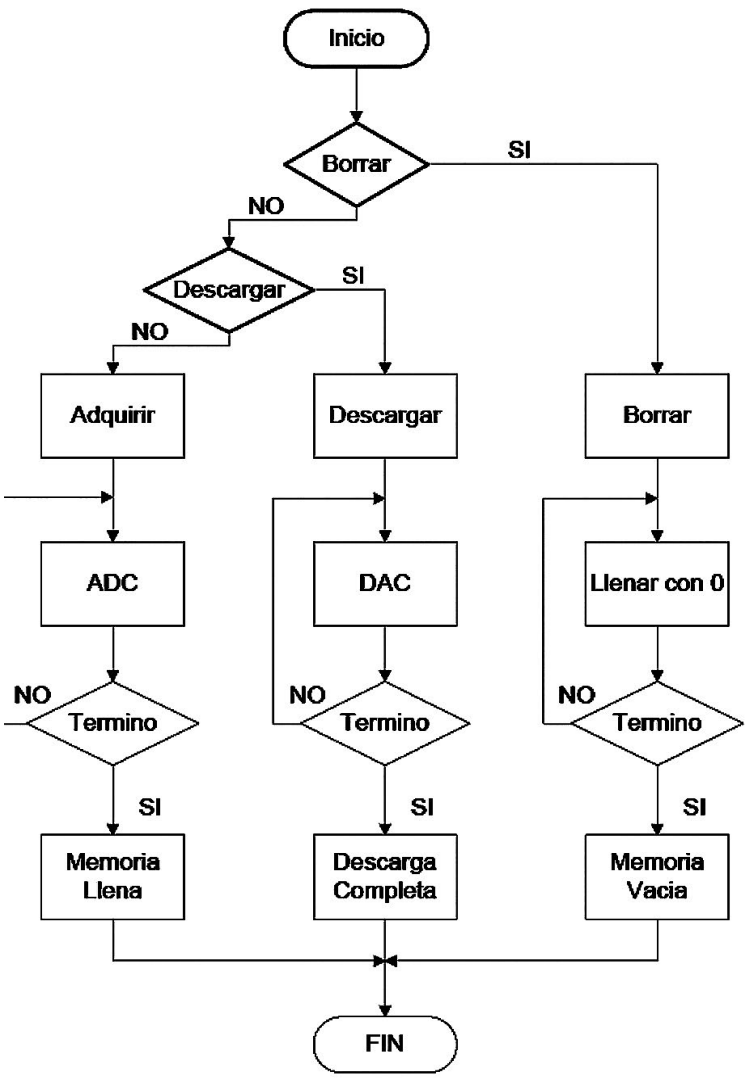

Fig. 7 Algoritmo de la programación del microcontrolador

\section{Construcción Del Prototipo Holter}

Se construyeron dos tarjetas electrónicas: una donde se procesan análogamente las derivaciones ECG y la otra donde se efectúa el proceso digital de las mismas. La Fig. 8, muestra la tarjeta análoga y la Fig. 9, la tarjeta digital.

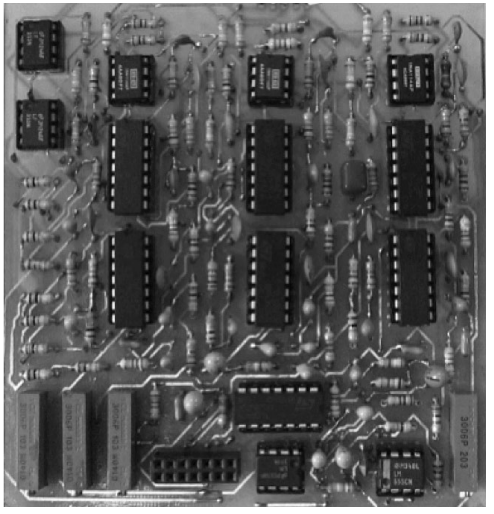

Fig. 8 Tarjeta de procesamiento análogo de las derivaciones ECG

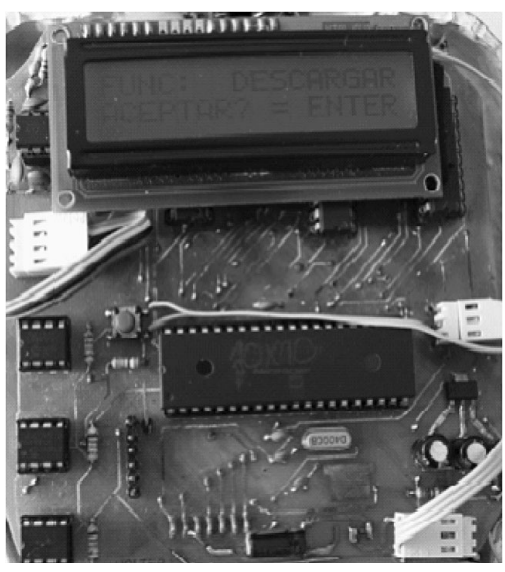

Fig. 9 Tarjeta de procesamiento digital de las derivaciones ECG

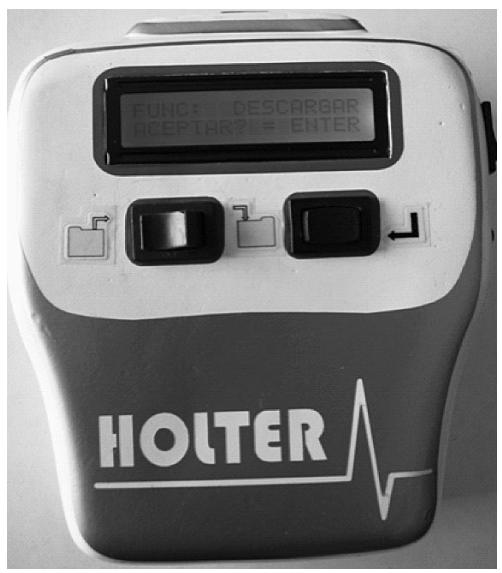

Fig. 10 Prototipo de equipo Holter de ECG

\section{ESPECIFICACIONES TÉCNICAS del Prototipo Holter}

Se muestran a continuación las especificaciones técnicas del prototipo Holter construido:

\section{A. Funcionales}

\begin{tabular}{|l|l|}
\hline Canales & 3 (DI, DII, Vx) \\
\hline Resolución & 8 bits \\
\hline Grabación 15 minutos & 10 segundos cada \\
\hline Interfaz de descarga & DAC0832 \\
\hline $\begin{array}{l}\text { Velocidad } \\
\text { de muestreo }\end{array}$ & $\begin{array}{l}138 \text { muestras/ } \\
\text { segundo }\end{array}$ \\
\hline Respuesta en frecuencia & $0,05 \mathrm{~Hz} \mathrm{a} \mathrm{40} \mathrm{Hz}$ \\
\hline $\begin{array}{l}\text { Factor de Rechazo } \\
\text { al MC> }\end{array}$ & $90 \mathrm{~dB}$ \\
\hline
\end{tabular}




\section{B. Memoria}

Duración de la grabación

24 horas

\begin{tabular}{|l|l|}
\hline Tipo & EEPROM 3I2C \\
\hline Referencia & 24LCS512 \\
\hline Capacidad & $512 \mathrm{~KB} /$ memoria \\
\hline
\end{tabular}

\section{Eléctricas}

\begin{tabular}{|l|l|}
\hline $\begin{array}{l}\text { Alimentación } \\
\text { a baterías }\end{array}$ & $\begin{array}{l}4 \times 1.5 \mathrm{~V} \mathrm{AAA} \\
(800 \mathrm{mAH})\end{array}$ \\
\hline Cable paciente & $\begin{array}{l}5 \text { broches } \\
\text { (RA,LA,RL,LL,Vx) }\end{array}$ \\
\hline Conector al Holter & PS2 \\
\hline
\end{tabular}

\section{Físicas}

\begin{tabular}{|l|l|}
\hline Dimensiones & $15 X 12.5 X 4 \mathrm{~cm}$ \\
\hline Peso con baterías & 320 gramos \\
\hline Carcaza & Fibra de vidrio \\
\hline
\end{tabular}

\section{Pruebas de Validación del Prototipo Holter}

Se llevaron a cabo dos tipos de pruebas de validación del prototipo Holter ECG: con simulador de paciente (Lion Heart) y con pacientes reales.

\section{A. Pruebas con simulador de paciente}

Se utilizó el simulador multiparamétrico de paciente Lion Heart 3, de la firma Biotek. Se probó con señales ECG normales y arrítmicas. Se almacenaron en el Holter y posteriormente se descargaron a un computador utilizando el sistema de instrumentación virtual Biopac MP150. La Fig. 11, muestra la conexión entre el Holter y el Lion Heart 3 en el proceso de adquisición y almacenamiento de las derivaciones ECG.

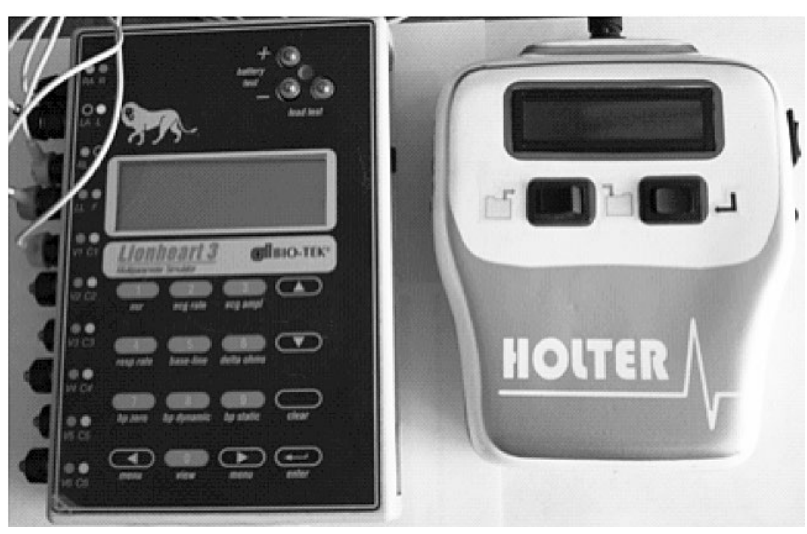

Fig. 11 Prueba del Holter ECG con el Simulador de Paciente $\mathrm{LH} 3$

La Fig. 12 muestra la descarga del Holter al computador lector a través del sistema Biopac.

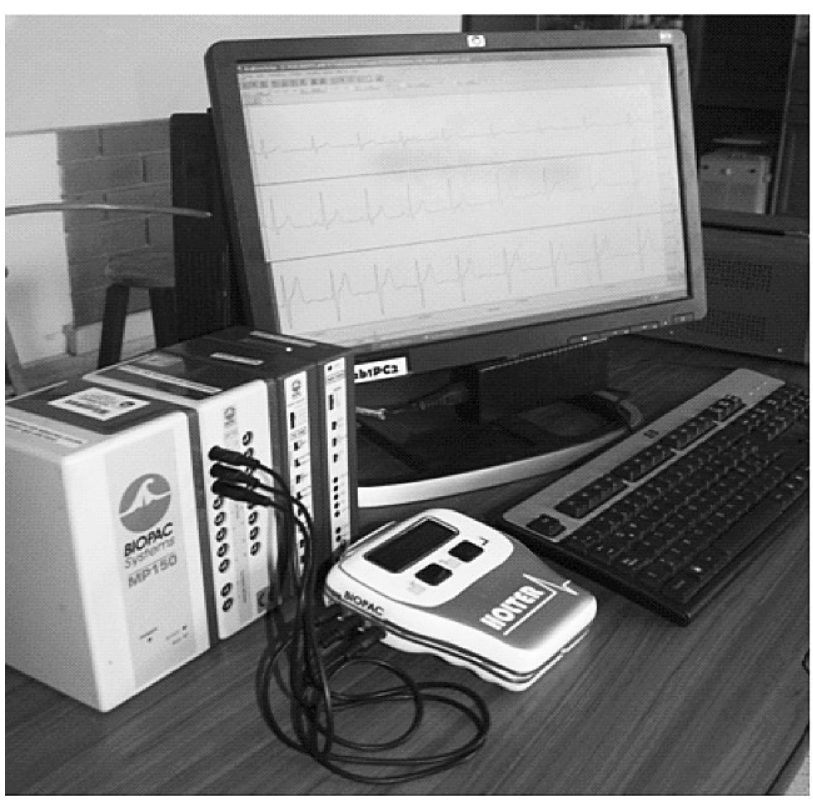

Fig. 12 Descarga del Holter al sistema Biopac

La Fig. 13 muestra las derivaciones ECG normales del simulador de paciente, adquiridas y descargadas del Holter. Se programó el simulador de paciente para que la derivación DI tuviera una amplitud máxima de $1 \mathrm{mV}$ y un ritmo cardiaco de 70 pulsos por minuto (ppm). 


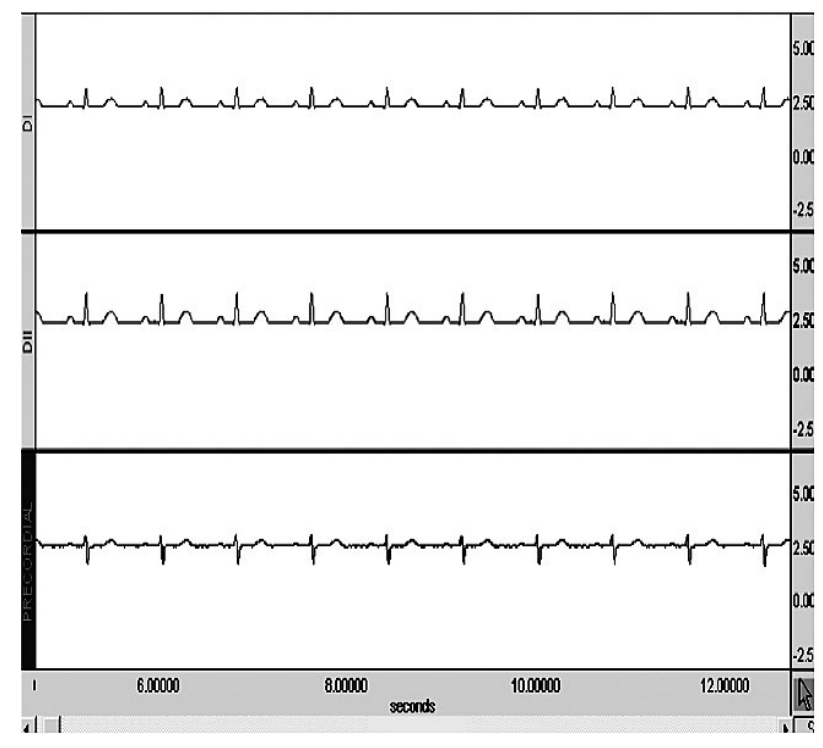

Fig. 13 Derivaciones ECG del simulador de paciente adquiridas y descargadas del Holter

La Fig. 14 muestra las derivaciones ECG normales, adquiridas del simulador de paciente directamente por el sistema Biopac.

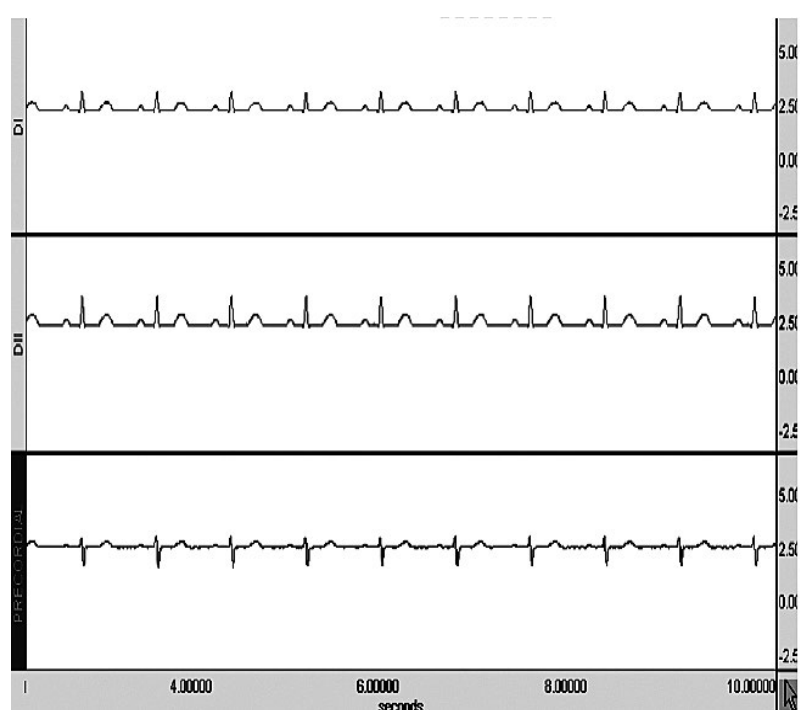

Fig. 14 Derivaciones ECG del simulador de paciente adquiridas directamente por el sistema Biopac

Comparando las figuras 13 y 14 se concluye que son las mismas, teniendo en cuenta que la amplitud máxima de la derivación DI, medida directamente con el Biopac es de 1V, y la adquirida a través del Holter es de $990 \mathrm{mV}$, ya que el canal de ECG del Holter tiene una ganancia de 1000 y el Biopac se programó para que multiplicara digitalmente las señales adquiridas por 1000. Además, el ritmo cardiaco medido en ambos casos fue de 70 ppm. Lo anterior indica que el prototipo Holter diseñado, no distorsiona las señales ECG, sino que las procesa con alta fidelidad.

Se simularon posteriormente diferentes tipos de arritmias con el Lion Heart 3, adquiriéndose y descargándose con el Holter, para examinar el comportamiento del mismo frente a estas patologías, puesto que estos equipos se utilizan principalmente en pacientes arrítmicos. La Fig. 15 muestra las derivaciones ECG arrítmicas, comprobándose la buena respuesta del equipo pues no distorsiona las señales ni en amplitud ni en frecuencia.

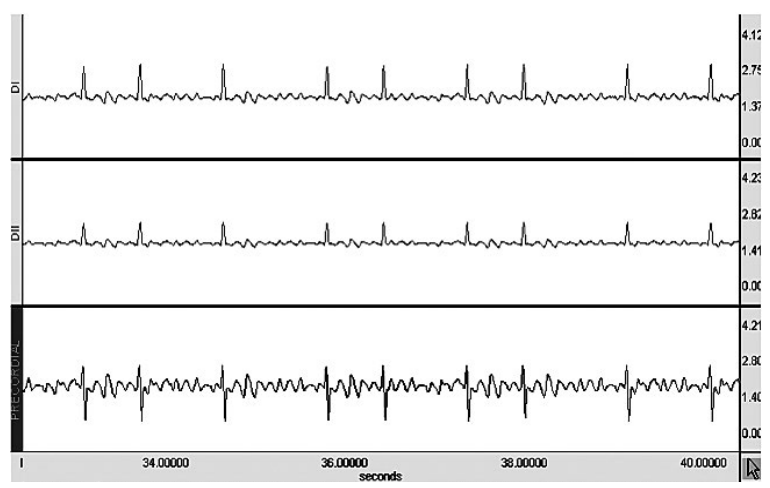

Fig. 15 Derivaciones ECG Arrítmicas

\section{B. Pruebas con pacientes reales}

La Fig. 16 muestra la conexión entre los electrodos ECG, colocados en el tórax del paciente, y el prototipo Holter, para adquirir las derivaciones DI, DIl y la precordial V2.

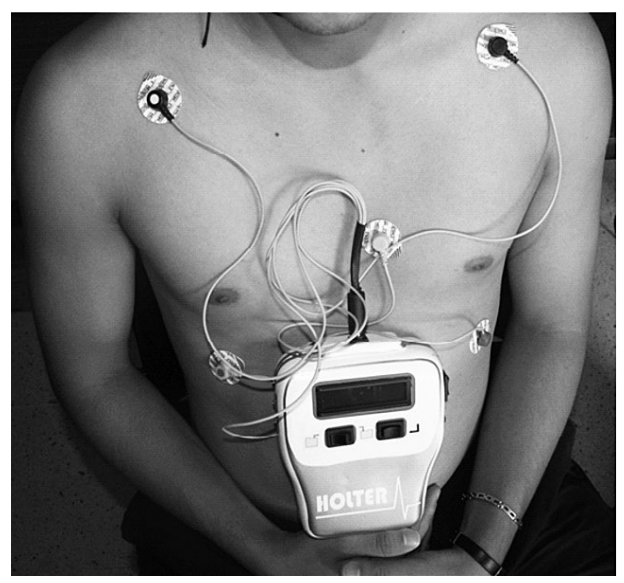

Fig. 16 Conexión del paciente al Holter 
Esta prueba mostró el comportamiento del prototipo Holter frente a los potenciales de semicelda DC, introducidos a las derivaciones ECG por los electrodos, que pueden producir recortamiento de las mismas debido a la saturación de los amplificadores.

La Fig. 17 muestra las derivaciones ECG del paciente, adquiridas y descargadas del Holter.

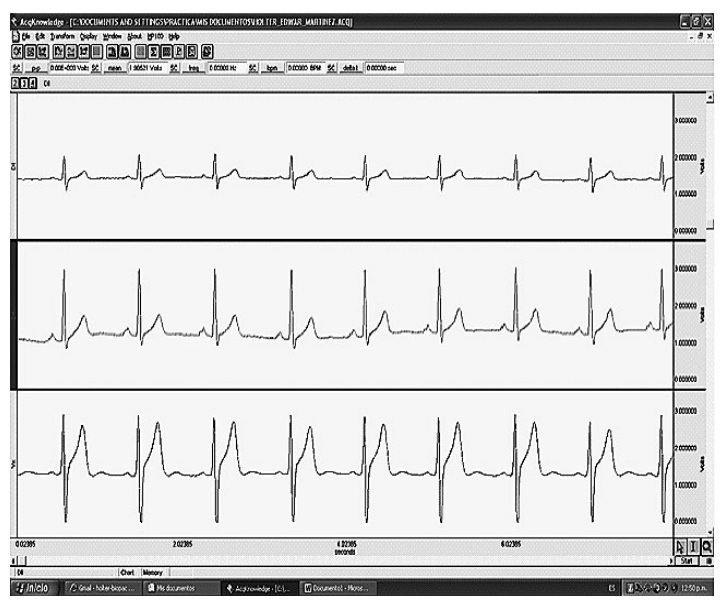

Fig. 17 Derivaciones ECG del paciente adquiridas y descargadas del Holter

Se puede observar que las tres derivaciones presentan un offset de aproximadamente $1.5 \mathrm{~V}$, sin que se recorten en sus picos máximos positivos y negativos.

La Fig. 18 muestra las derivaciones ECG adquiridas del paciente directamente por el sistema Biopac.

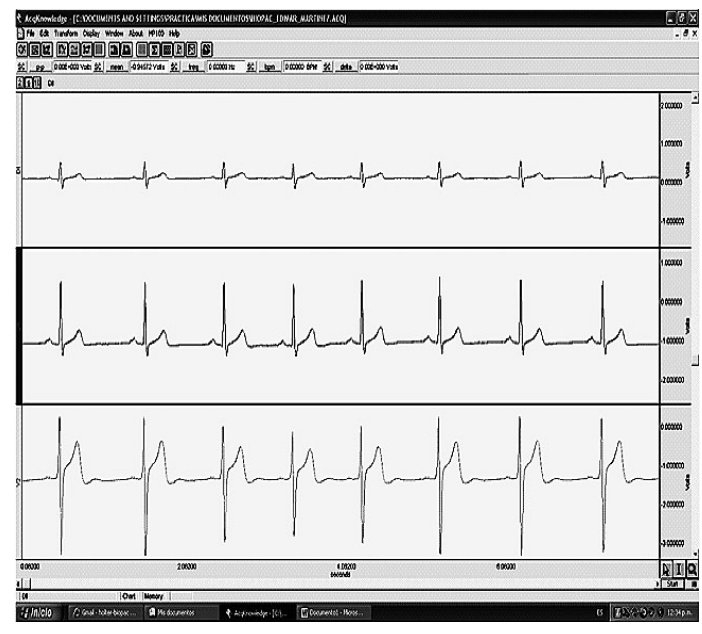

Fig. 18 Derivaciones ECG del paciente adquiridas directamente por el sistema Biopac
Comparando las figuras 17 y 18 se concluye que son las mismas, pues la correlación de los valores pico-pico es del $99 \%$ y en los intervalos entre las ondas $\mathrm{R}$ que determinan el ritmo cardiaco es de $100 \%$. Esto confirma, nuevamente, que el prototipo Holter diseñado, no distorsiona las señales ECG, sino que las procesa con alta fidelidad.

\section{ConcLusiones}

Se logró construir un prototipo de Holter ECG que entregara las derivaciones almacenadas en formato analógico, para ser adquiridas por cualquier sistema de instrumentación virtual, sin la necesidad de los protocolos de comunicación, propios de la transmisión digital.

El prototipo construido elimina el ruido de 60 $\mathrm{Hz}$ introducido en cualquier señal por la red de potencia eléctrica y, por lo tanto, facilita el estudio y análisis por parte del cardiólogo al no presentar interferencias.

\section{ReCOMENDACIONES}

Se recomienda que a partir de este prototipo construido, se construya un equipo comercial utilizando componentes de montaje superficial, que permita reducir el tamaño del mismo y el consumo de las baterías.

Se sugiere iniciar el desarrollo de un software que permita el análisis de forma automática de las derivaciones ECG, para reducir sustancialmente el tiempo de estudio por parte del cardiólogo y presentar resultados más exactos. 


\section{Referencias}

[1] C. Muller, S. Wong y A. La Cruz., " Prototipo de Unidad de Registro EDF Para Holter Digital de Bajo Costo," IFMBE Proceedings, vol. 18, pp. 480-483,2001.

[2] D. J. Jiménez, R. Guardurrama, R.I Gonzalez., " Diseño de un Registrador para Pruebas de Holter" presentado en el VII Congreso de la Sociedad Cubana de Bioingeniería, Habana, Cuba, 2007.

[3] J. L. Palma., Electrocardiografía de Holter, vol. I. Madrid, Norma, p. 143-173, 1983.

[4] L. M. Jadraque., Cardiopatía Isquémica, vol. I. Madrid: Norma, p. 177, 1988.

[5] G. Montes de Oca, G. Rodriguez, R. Almeida., "Analizador de un Sistema de Monitoreo Holter de 3 canales: Excorde 3C" presentado en el VII Congreso de la Sociedad Cubana de Bioingeniería, Habana, Cuba, 2007. 